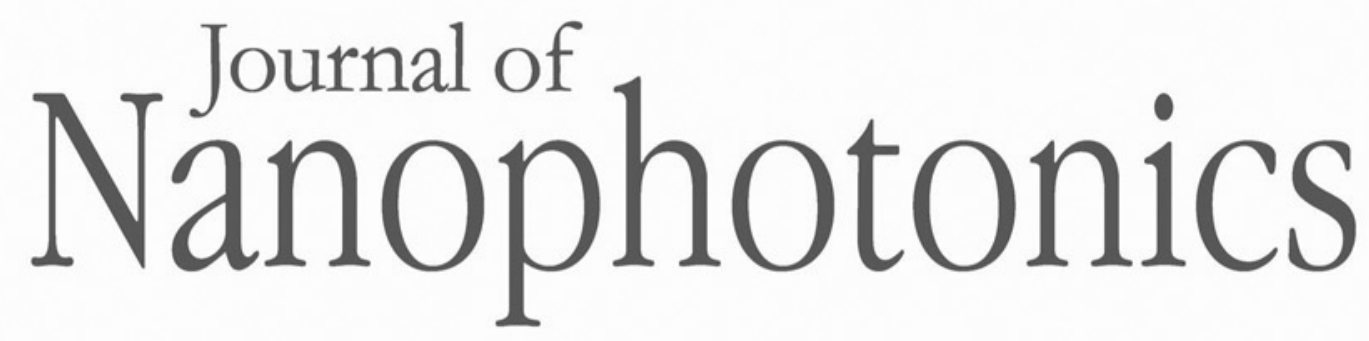

\title{
Aggregation of nanoparticles in endosomes and lysosomes produces surface-enhanced Raman spectroscopy
}

Leanne J. Lucas

Xiaoke K. Chen

Aaron J. Smith

Mladen Korbelik

Haishan Zeng

Patrick W. K. Lee

Kevin Cecil Hewitt 


\title{
Aggregation of nanoparticles in endosomes and lysosomes produces surface-enhanced Raman spectroscopy
}

\author{
Leanne J. Lucas, ${ }^{a}$ Xiaoke K. Chen, ${ }^{\text {b }}$ Aaron J. Smith, ${ }^{\text {a }}$ Mladen Korbelik, \\ Haishan Zeng, ${ }^{\mathrm{c}}$ Patrick W. K. Lee, ${ }^{\mathrm{d}}$ and Kevin Cecil Hewitt ${ }^{\mathrm{a}, *}$ \\ ${ }^{a}$ Dalhousie University, Department of Physics and Atmospheric Science, Halifax, \\ Nova Scotia, B3H 4R2, Canada \\ ${ }^{\mathrm{b}}$ Simon Fraser University, Department of Physics, Burnaby, British Columbia, V5A 1S6, Canada \\ ${ }^{c} \mathrm{BC}$ Cancer Agency Research Centre, Imaging Unit-Integrative Oncology, Vancouver, \\ British Columbia, V5Z 1L3, Canada \\ ${ }^{\mathrm{d}}$ Dalhousie University, Department of Microbiology and Immunology, Halifax, \\ Nova Scotia, B3H 4R2, Canada
}

\begin{abstract}
The purpose of this study was to explore the use of surface-enhanced Raman spectroscopy (SERS) to image the distribution of epidermal growth factor receptor (EGFR) in cells. To accomplish this task, 30-nm gold nanoparticles (AuNPs) tagged with antibodies to EGFR $\left(10^{12}\right.$ per $\left.\mathrm{mL}\right)$ were incubated with cells $\left(10^{6}\right.$ per $\left.\mathrm{mL}\right)$ of the A431 human epidermoid carcinoma and normal human bronchial epithelial cell lines. Using the 632.8-nm excitation line of a He-Ne laser, Raman spectroscopy measurements were performed using a point mapping scheme. Normal cells show little to no enhancement. SERS signals were observed inside the cytoplasm of A431 cells with an overall enhancement of 4 to 7 orders of magnitude. Raman intensity maps of the 1450 and $1583 \mathrm{~cm}^{-1}$ peaks correlate well with the expected distribution of EGFR and AuNPs, aggregated following uptake by endosomes and lysosomes. Spectral features from tyrosine and tryptophan residues dominate the SERS signals. () The Authors. Published by SPIE under a Creative Commons Attribution 3.0 Unported License. Distribution or reproduction of this work in whole or in part requires full attribution of the original publication, including its DOI. [DOI: 10.1117/1.JNP.9.093094]
\end{abstract}

Keywords: surface-enhanced Raman spectroscopy; epidermal growth factor receptor overexpression; gold nanoparticles; cancer imaging; endosomes; lysosomes.

Paper 14107P received Sep. 27, 2014; accepted for publication Dec. 29, 2014; published online Jan. 23, 2015.

\section{Introduction}

A number of cancers are characterized by overexpression of the epidermal growth factor receptor (EGFR), a membrane protein which mediates cell growth, proliferation, and differentiation in multiple tissues. Many epithelial tumors have been found to express high numbers of EGF receptors, ${ }^{1-7}$ and the receptor levels are associated with poor clinical prognosis in cancers of the bladder, ${ }^{4}$ lung, ${ }^{5}$ and breast. ${ }^{4,6}$ Antibodies for EGFR tagged with fluorescent probes have been used as contrast agents to image EGFR overexpression. ${ }^{8}$ The EGFR-induced shift in the plasmon resonance has also been used as an image contrast method. ${ }^{9}$ Both of these methods provide at most a 10 -fold intensity contrast factor.

To overcome the limited contrast, we explore the use of surface-enhanced Raman spectroscopy (SERS). By exciting a sample with laser light, energy is transferred to (Stokes process) or lost (anti-Stokes process) by the medium in an inelastic scattering process referred to as Raman spectroscopy. The scattered light is a fingerprint of the set of atomic vibrations, which is unique to the arrangement and types of atoms under study. In general, 1 in $10^{6}$ photons is scattered inelastically in this manner. By tuning the incident laser frequency to the plasma frequency of the nanoparticle assembly, an overall enhancement of 7 to 8 orders of magnitude of the

*Address all correspondence to: Kevin Cecil Hewitt, E-mail: Kevin.Hewitt@Dal.ca 
scattered signal is possible; ${ }^{10}$ this is referred to as the surface-enhanced Raman scattering effect. Over the past few years, there has been a flurry of activity in the application of Raman spectroscopy to problems in biology. Sha et al. ${ }^{11}$ have reviewed the field of the application of SERS to oncology. The idea of using SERS to image cancerous tissue was exploited by Kah et al. ${ }^{12} \mathrm{~A}$ self-assembled SERS-active, gold nanoparticle (AuNP) monolayer film tagged with anti-EGFR was used to distinguish cancerous and normal cells in saliva for early diagnosis of oral cancer. Qian et al. ${ }^{13}$ have also employed the SERS to target EGFR overexpressing cells in vivo using mouse xenograft tumor models. Approximately 1.4 to $1.5 \times 10^{4}$ reporter molecules (e.g., malachite green) are adsorbed on each 60 -nm gold particle and stabilized with $3.0 \times 10^{4}$ thiol-polyethyleneglycol (thiol-PEG). Their SERS particles exhibit a signal that is 2 orders of magnitude larger than quantum dots, highlighting the power of the technique. Keren et al. ${ }^{14}$ have demonstrated the multimodal SERS detection using antibody-tagged Oxonica nanoparticles. Kim et al. ${ }^{15}$ developed the antibody-conjugated, silver nanoparticles-embedded silica spheres to target HER2 and CD10 on cellular membrane constructs. None of these reports have mapped the distribution and fate of the nanoparticles within a single cell. Our study is an extension of an earlier contribution ${ }^{16}$ and aims to determine the microscopic distribution of antibody-tagged AuNPs in order to map the cellular distribution of EGFR in both normal and cancerous cell cultures.

\section{Materials and Methods}

\subsection{Gold Nanoparticle Synthesis}

The AuNPs used in the experiment were synthesized using the well-known method of Turkevich et al. ${ }^{17}$ and Frens. ${ }^{18}$ A $0.01 \%$ by weight gold chlorate solution was heated with stirring to boiling in an extremely clean round bottomed flask fitted with a refluxer in a fume hood. The flask was heated in a bath of silicon oil to ensure even heat distribution. A precise volume of $1 \%$ by weight sodium citrate was added to reduce the gold salt with continued boiling. The amount of sodium citrate added to the stirring gold solution ultimately determined the final size of the AuNPs, with smaller volumes of citrate producing larger particles.

\subsection{Gold Nanoparticle Characterization}

Three techniques were used to characterize the AuNPs and their derivatives: UV-visible spectroscopy (UV-Vis), transmission electron microscopy (TEM), and dynamic light scattering (DLS). From UV-Vis spectroscopy, the approximate size, plasmon absorbance wavelength, and concentration of the nanoparticles were obtained. The UV-Vis analysis was performed using a UV-1700 (Shimadzu) or ND-1000 (Nanodrop) spectrophotometer. The concentration of nanoparticles can be calculated using the absorbance maximum:

$$
A=\frac{N \sigma b c}{\ln 10},
$$

where $A$ is the absorbance (unitless), $N$ is Avogadro's number $\left(6.02 \times 10^{23}\right.$ molecules/mole), $\sigma$ is the extinction cross-section $\left(\mathrm{cm}^{2}\right), b$ is the path length $(\mathrm{cm})$, and $c$ is the nanoparticle concentration (NPs $/ \mathrm{mL}$ ). The extinction cross-section for 30-nm AuNPs is $3 \times 10^{-11} \mathrm{~cm}^{2}$. The TEM was used to determine the particle size distribution. Approximately 10 to $20 \mu \mathrm{L}$ of the nanoparticle solution was pipetted onto a copper backed carbon TEM grid and allowed to air dry overnight. A Hitachi H-8000 TEM, operating at $200 \mathrm{kV}$, was used to image the nanoparticles at a magnification of $120000 \times$. The DLS was also used to characterize the particle size distribution. An ALV DLS/SLS apparatus was used to measure the samples. Once the diffusion coefficient $(D)$ is known, the radius $(R)$ of the particles can be calculated using the StokesEinstein equation: $D=k_{\mathrm{B}} T / 6 \pi \eta R$, where $k_{\mathrm{B}}$ is the Boltzmann constant, $T$ is the temperature in Kelvin, and $\eta$ is the viscosity.

\subsection{Antibody Conjugation to Gold Nanoparticles}

To test the effectiveness of anti-EGFR antibodies, a series of experiments were run. Antibodies specific for human EGFR, produced in mice (Sigma-Aldrich, E2156, Monoclonal anti-EGFR- 
human, clone 225, purified mouse immunoglobin, Immunogen-partially purified EGF receptors from human A431 cells), IgG polyclonal nonspecific antibodies (Sigma Aldrich, I8765, Mouse IgG-technical grade from normal mouse serum), and antibody free solutions were incubated with nanoparticles. AuNPs of approximately $1.4 \times 10^{10} \mathrm{NP} / \mathrm{mL}$ were diluted in a $1: 1$ ratio with $50 \mathrm{mM}$ HEPES and then centrifuged at $3000 \mathrm{rcf}$ for $1 \mathrm{~h}$ at $4^{\circ} \mathrm{C}$ to concentrate. This gives an approximate volume of $4 \mathrm{~mL}$ and concentration of $1.4 \times 10^{11} \mathrm{NP} / \mathrm{mL}$. A solution of antibodies at a final concentration of $4.75 \mu \mathrm{g} / \mathrm{mL}$ was prepared in $25 \mathrm{mM}$ HEPES. This solution was added to the nanoparticle solution. An equal volume of $0.4 \mathrm{mg} / \mathrm{mL}$ PEG solution was added dropwise. The solution was incubated overnight at $4^{\circ} \mathrm{C}$ or until use. Unattached antibodies were removed by centrifuging at $3000 \mathrm{rcf}$ for $1 \mathrm{~h}$ at $4^{\circ} \mathrm{C}$ just prior to use. The solution was resuspended in $50 \mathrm{mM}$ HEPES, giving an approximate volume of $1 \mathrm{~mL}$. This solution was then characterized with UV-Vis. A shift of 6 to $10 \mathrm{~nm}$ indicates that the antibodies are attached. The approximate concentrations of the components in $1 \mathrm{~mL}$ of the prepared solution were: nanoparticles $-5 \times 10^{11} \mathrm{NP} / \mathrm{mL}$, antibodies $-4.75 \mu \mathrm{g} / \mathrm{mL}$, and PEG- $0.2 \mathrm{mg} / \mathrm{mL}$. The antibody-free, or bare nanoparticle, solutions were subjected to the same procedure to account for any losses.

\subsection{Cell Preparation}

Two types of cells were used: EGFR overexpressing human epidermoid carcinoma A431 cell line from ATCC and normal human bronchial epithelial (NHBE) cells from Lonza. The antibody-conjugated AuNPs $\left([\mathrm{AuNP}]=10^{12}\right.$ per $\mathrm{mL}$ ) were then attached to the cells $([\mathrm{cells}]=$ $10^{6}$ per $\mathrm{mL}$ ), leading to an approximate ratio of $10^{6}$ AuNPs/cell. In a biosafety cabinet, the prepared antibody solution was pipetted into a dish of cells where the cells had been grown on a coverslip. The cells were counted to give no more than 0.5 million for an experiment. The solution was incubated with the cells for $30 \mathrm{~min}$ and washed with sterile $50 \mathrm{mM}$ HEPES. The coverslip was flipped into a clean glass bottomed Petri dish. A polymer spacer was pressed against the coverslip to secure it. The dish was filled with HEPES buffer ( $\mathrm{pH}$ 7.4) and loaded into a custom-made Raman sample holder.

\subsection{Raman Spectra}

Raman spectra were obtained with a LabRam confocal micro-Raman system (Jobin Yvon Inc., Edison, New Jersey), which consists of a grating spectrograph coupled to an Olympus BX40 microscope with a motorized $x-y$ stage. A 632.8-nm He-Ne laser excited the Raman spectra by focusing through a $100 \times$ objective with a coverslip correction collar. The incident power at the sample is $200 \mu \mathrm{W}$. The Raman scattered light was collected in a confocal arrangement by the same objective and spectrally dispersed using a Horiba Jobin Yvon Raman Spectrometer onto a thermoelectrically cooled CCD detector. The system also has a video system for white light image capture, a computer to collect and process data, and a motorized stage for translation along $x, y$, and $z$.

The system was calibrated with the silicon line at $520.7 \mathrm{~cm}^{-1}$ (at least 2000 and up to 9000 counts/s of signal was achieved). A grating of 1800 grooves $/ \mathrm{mm}$, a confocal pinhole of $400 \mu \mathrm{m}$, and an input slit width of $200 \mu \mathrm{m}$ were used. Spectra were collected with a $1 \mathrm{~s}$ integration time, averaged over two scans. A step size of $1 \mu \mathrm{m}$ or less was utilized, typically $0.3 \mu \mathrm{m}$, to map the spectral features of a cell. In this confocal arrangement, the depth resolution is $2 \mu \mathrm{m}$. The intensity distribution in a narrow spectral range was used to produce a Raman map of the cell of interest.

\section{Results and Discussion}

\subsection{Nanoparticle Preparation and Characterization}

To produce gold colloidal solutions, $1 \mathrm{~mL}$ of $1 \%$ by weight sodium citrate was added dropwise to a boiling solution of $100 \mathrm{~mL} 0.01 \%$ (by weight) gold salt at $100^{\circ} \mathrm{C}$. The reaction is complete 
when the solution turns a reddish color (in 5 to $10 \mathrm{~min}$ ). The gold colloidal solution was characterized by UV-Vis spectroscopy, TEM, and DLS. An analysis of the TEM images in Fig. 1(a) reveals particle diameters of approximately $30 \pm 5 \mathrm{~nm}$, while the DLS histogram [Fig. 1(b)] implies an average particle size of approximately $34 \mathrm{~nm}$. An increase is expected since DLS measures the hydrodynamic radius including the citrate capping molecule. UV-Vis absorption [Fig. 1(c)] exhibits a maximum absorption at $532 \mathrm{~nm}$ for the bare nanoparticles (blue curve) and $536 \mathrm{~nm}$ for AuNPs tagged with anti-EGFR (pink curve). The latter curve is obtained after eliminating unattached anti-EGFR by centrifugation at $3000 \mathrm{rcf}$. The 4-nm red-shift between the maxima of the absorption spectra is consistent with the attachment of anti-EGFR to the AuNPs, which is ultimately due to the increase in the index of refraction of the medium surrounding the AuNPs.

\subsection{Anti-EGFR-Tagged Gold Nanoparticles}

The anti-EGFR-tagged AuNPs were aggregated by adding 0.01 M PBS (pH 7.4) to the gold colloidal solution. Figure 2 is the Raman spectra of the point indicated in the optical image of an aggregate (inset). The data were highly reproducible if the laser power, under the

(a)

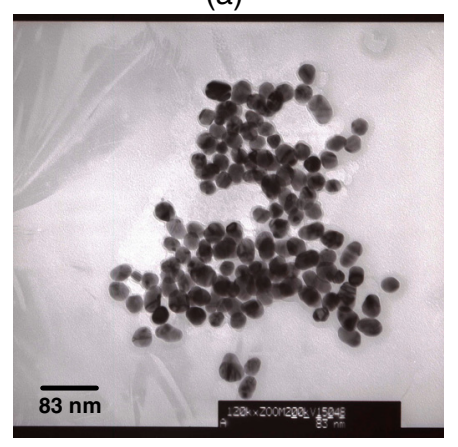

(b)

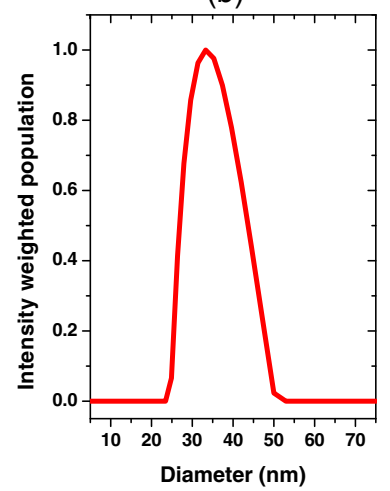

(c)

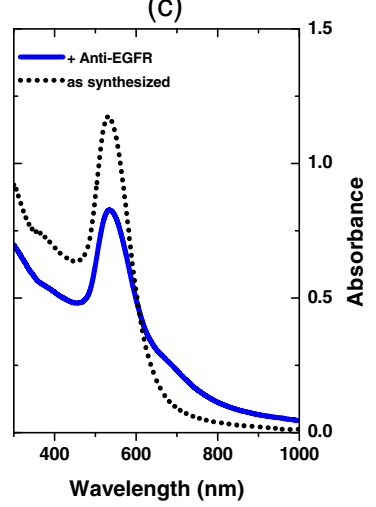

Fig. 1 TEM images (a) and corresponding DLS histogram (b) of bare gold nanoparticles (LLB47B) and (c) UV-Vis absorption spectra of bare (black dotted curve, LLB47B) and anti-EGFR-tagged AuNPs (solid blue curve, LLB53A).

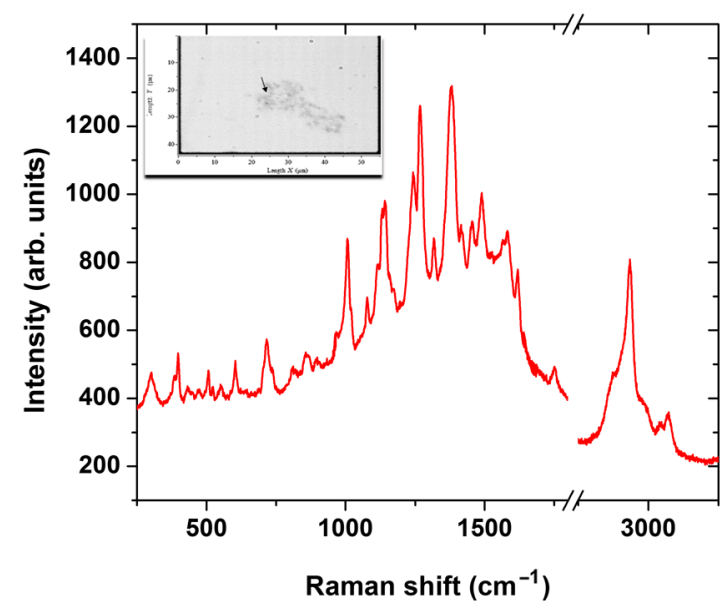

Fig. 2 SERS spectra obtained from overlapping five spectral ranges. Spectra were obtained at the point indicated by the arrow in the inset with a 1-s collection time, averaged over two scans. A map across the entire aggregate exhibited highly reproducible spectra. White light image of anti-EGFRlabeled nanoparticles (LLA141A) is shown in the inset. 
Lucas et al.: Aggregation of nanoparticles in endosomes and lysosomes produces surface-enhanced...

Table 1 Peak positions, relative strength, and tentative assignments for the SERS spectra of antiEGFR. Peak positions obtained from Lorentzian fits (very strong-vs, strong-s, moderate-m, weak-w, and very weak-vw).

\begin{tabular}{|c|c|c|}
\hline $\begin{array}{l}\text { Raman } \\
\text { shift }\left(\mathrm{cm}^{-1}\right)\end{array}$ & $\begin{array}{l}\text { Relative peak } \\
\text { intensity }\end{array}$ & Assignment \\
\hline $\begin{array}{l}282 \pm 1 \\
299.2 \pm 0.8\end{array}$ & $\begin{array}{c}v w \\
s\end{array}$ & \}S-Au plus torsional \\
\hline $313 \pm 3$ & vw & \\
\hline $367 \pm 3$ & vw & O-Au plus torsional \\
\hline $\begin{array}{l}383.6 \pm 0.3 \\
397.8 \pm 0.1\end{array}$ & $\begin{array}{c}\mathrm{m} \\
\mathrm{s}\end{array}$ & \}N-Au plus torsional \\
\hline $432 \pm 1$ & vw & Ring bending/torsional mode \\
\hline $449.9 \pm 0.9$ & vw & Ring torsion of phenyl ${ }^{20}$ \\
\hline $467 \pm 1$ & vw & Valine or glutamic acid ${ }^{21}$ \\
\hline $475.0 \pm 0.9$ & vw & Valine or glutamic acid ${ }^{21}$ \\
\hline $496 \pm 2$ & vw & \\
\hline $\begin{array}{l}506.6 \pm 0.2 \\
523.4 \pm 0.3\end{array}$ & $\begin{array}{c}\mathrm{m} \\
\mathrm{vw}\end{array}$ & \}S-S stretching vibrations in cysteine residues ${ }^{19,20}$ \\
\hline $551.5 \pm 0.5$ & w & Tryptophan, histidine, or arginine ${ }^{21}$ \\
\hline $603.8 \pm 0.2$ & $\mathrm{~m}$ & C-S stretch glycine, glutamate, or phenylalanine ${ }^{21}$ \\
\hline $639 \pm 1$ & $\mathrm{vw}$ & $\mathrm{C}-\mathrm{S}$ stretch and $\mathrm{C}-\mathrm{C}$ twist of tyrosine $\mathrm{e}^{20}$ \\
\hline $703 \pm 1$ & w & C-C twist of tyrosine or phenylalanine $e^{20}$ \\
\hline $716.1 \pm 0.2$ & $\mathrm{~m}$ & Deformation vibrations of $\mathrm{COO}^{-}$(Ref. 19) \\
\hline $737.4 \pm 0.6$ & w & $\mathrm{C}-\mathrm{S}$ stretch ${ }^{20}$ or deformation vibrations of $\mathrm{COO}^{-}$(Ref. 19) \\
\hline $812.6 \pm 0.5$ & vw & Tyrosine ring breathing vibrations (exposed) ${ }^{22}$ \\
\hline $856.9 \pm 0.9$ & w & Tyrosine ring breathing vibrations (buried) ${ }^{19,22}$ \\
\hline $870.2 \pm 0.6$ & $\mathrm{vw}$ & $\mathrm{C}-\mathrm{C}$ stretch in proteins ${ }^{20}$ \\
\hline $896.7 \pm 0.5$ & w & C-C stretch in glycine ${ }^{19}$ \\
\hline $947 \pm 4$ & vw & C-C stretch proline and valine $\mathrm{e}^{20}$ \\
\hline $1007.1 \pm 0.2$ & vs & Ring $\mathrm{C}-\mathrm{C}$ stretch in phenylalanine or tryptophan ${ }^{19}$ \\
\hline $1077.9 \pm 0.3$ & w & Tryptophan or $\mathrm{C}-\mathrm{N}$ stretching mode of proteins ${ }^{20}$ \\
\hline $1114.0 \pm 0.4$ & w & $\mathrm{C}-\mathrm{C}$ vibrations \\
\hline $1129 \pm 0.2$ & vs & Symmetric $\mathrm{C}-\mathrm{C}$ vibrations ${ }^{19}$ \\
\hline $1141.2 \pm 0.5$ & $\mathrm{~m}$ & Symmetric $\mathrm{C}-\mathrm{C}$ vibrations ${ }^{19}$ \\
\hline $1240.2 \pm 0.4$ & s & Tryptophan ring vibrations ${ }^{19}$ \\
\hline $1269.1 \pm 0.2$ & vs & $\mathrm{C}-\mathrm{H}$ bending mode \\
\hline $1317.1 \pm 0.4$ & w & Carbonyl symmetric stretch \\
\hline $1379.6 \pm 0.1$ & vs & Carbonyl symmetric stretch \\
\hline $1422.0 \pm 0.5$ & w & $\mathrm{COO}^{-}$stretch in IgG aspartic or glutamic acid or $\mathrm{C}=\mathrm{C}$ stretch $^{20}$ \\
\hline $1454.2 \pm 0.5$ & $\mathrm{~m}$ & $\mathrm{CH}_{2}$ deformations ${ }^{22}$ \\
\hline
\end{tabular}


Table 1 (Continued).

\begin{tabular}{lcl}
\hline \hline $\begin{array}{l}\text { Raman } \\
\text { shift }\left(\mathrm{cm}^{-1}\right)\end{array}$ & $\begin{array}{c}\text { Relative peak } \\
\text { intensity }\end{array}$ & \multicolumn{1}{c}{ Assignment } \\
\hline $1490.5 \pm 0.6$ & $\mathrm{~m}$ & Tryptophan, tyrosine, ring stretch/breathing ${ }^{23}$ \\
$1552 \pm 3$ & vw & $\mathrm{C}-\mathrm{C}$ stretch of tryptophan ${ }^{23}$ \\
$1584.5 \pm 0.3$ & w & Asp, glu, C=O stretch ${ }^{23}$ \\
$1619.6 \pm 0.2$ & w & $\mathrm{C}=\mathrm{C}$ stretch of tryptophan or phenylalanine ${ }^{20}$ \\
$1749.2 \pm 0.4$ & w & $\mathrm{C}=\mathrm{O}$ stretch of aspartic acid ${ }^{23}$ \\
$2878 \pm 1$ & w & $\mathrm{CH}{ }_{2}$ asymmetric stretch of proteins ${ }^{20}$ \\
$2934.3 \pm 0.1$ & vs & $\mathrm{C}-\mathrm{H}$ vibrations in proteins; Chain end $\mathrm{CH}_{3}$ symmetric band \\
$2988.6 \pm 0.7$ & w & $\mathrm{C}-\mathrm{H}$ stretching \\
$3043 \pm 1$ & vw & $\mathrm{C}-\mathrm{H}$ stretching \\
$3071.4 \pm 0.4$ & vw & $\mathrm{C}-\mathrm{H}$ stretching \\
\hline \hline
\end{tabular}

$100 \times$ objective, is kept below $200 \mu \mathrm{W}$. The spectral features are specific to the attached antibody. Huang et al. ${ }^{19}$ reported the SERS spectra of anti-EGFR. The peaks reported by them are comparable with those found here (Table 1). The absence of an amide I band near $1670 \mathrm{~cm}^{-1}$ is striking, as this feature is normally prominent in the nonSERS Raman spectra of proteins. This band requires the presence of the entire backbone in the enhancement area in a parallel orientation. Its absence implies that only the side chain groups were enhanced.

Proteins are expected ${ }^{23}$ to bind to the nanoparticles through those amino acids which form complexes with gold; among them sulfur containing cysteine, nitrogen containing histidine and tryptophan, and the basic amino acids lysine and arginine, at high $\mathrm{pH}$ above their isoelectric point (pI). The acids, aspartic and glutamic acids, would also be expected to adsorb when deprotonated, as would tyrosine through the oxygen atom side group following the loss of hydrogen. At the $\mathrm{pH}$ used in these experiments, 7.4, the deprotonated acids would be expected to bind to the nanoparticles, but not the bases. The results outlined in Table 1 confirm this hypothesis. The tentative peak assignments are taken from a variety of sources. Cysteine, histidine, tryptophan,

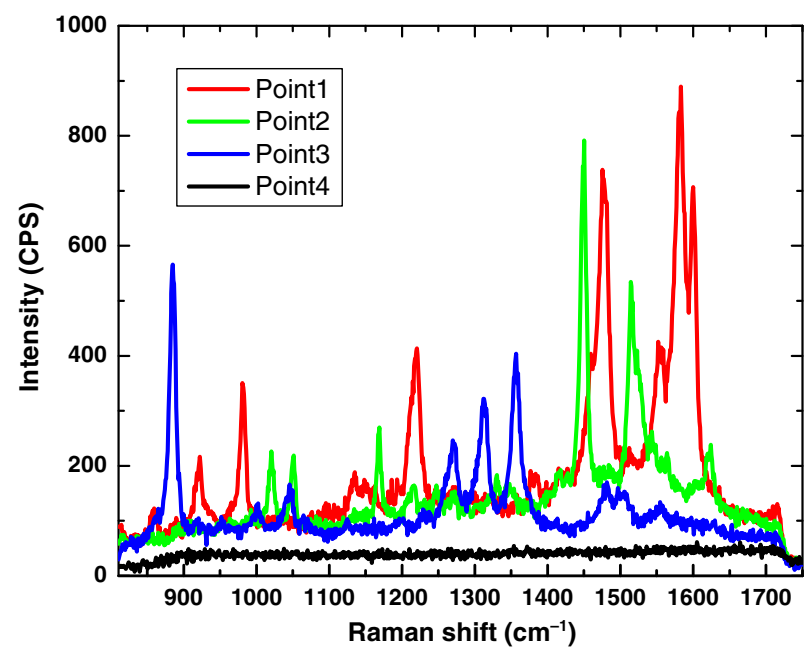

Fig. 3 SERS spectra taken from four locations inside and outside the A431 cell indicated in Fig. 4(a). The intensity is in units of counts per second (CPS). 
tyrosine, aspartate, and glutamate peaks dominate the spectra below $1800 \mathrm{~cm}^{-1}$, while the highfrequency peaks are likely due to $\mathrm{C}-\mathrm{H}$ and $\mathrm{CH}_{3}$ vibrations of these amino acids. In addition, among these amino acids, few characteristic peaks appear below $400 \mathrm{~cm}^{-1}$. The appearance of low-frequency features arises from the binding of sulfur, nitrogen, and oxygen to the AuNP; for which the magnitude of charge transfer decreases in the same order, sulfur-nitrogen-gold. This has the effect of reducing the contribution of charge-transfer effects to the resonance enhancement in SERS, reducing the strength of these modes, in the order of 302,397 , and $386 \mathrm{~cm}^{-1}$.

Six modes exhibit very strong enhancement, corresponding to vibrational frequencies of $1009 \mathrm{~cm}^{-1}$ (phenylalanine or tryptophan), $1131 \mathrm{~cm}^{-1}$ (symmetric C-C stretch), $1267 \mathrm{~cm}^{-1}$ (C-H bending modes), $1380 \mathrm{~cm}^{-1}$ (carbonyl symmetric stretch), and $2935 \mathrm{~cm}^{-1}(\mathrm{C}-\mathrm{H}$ stretch).
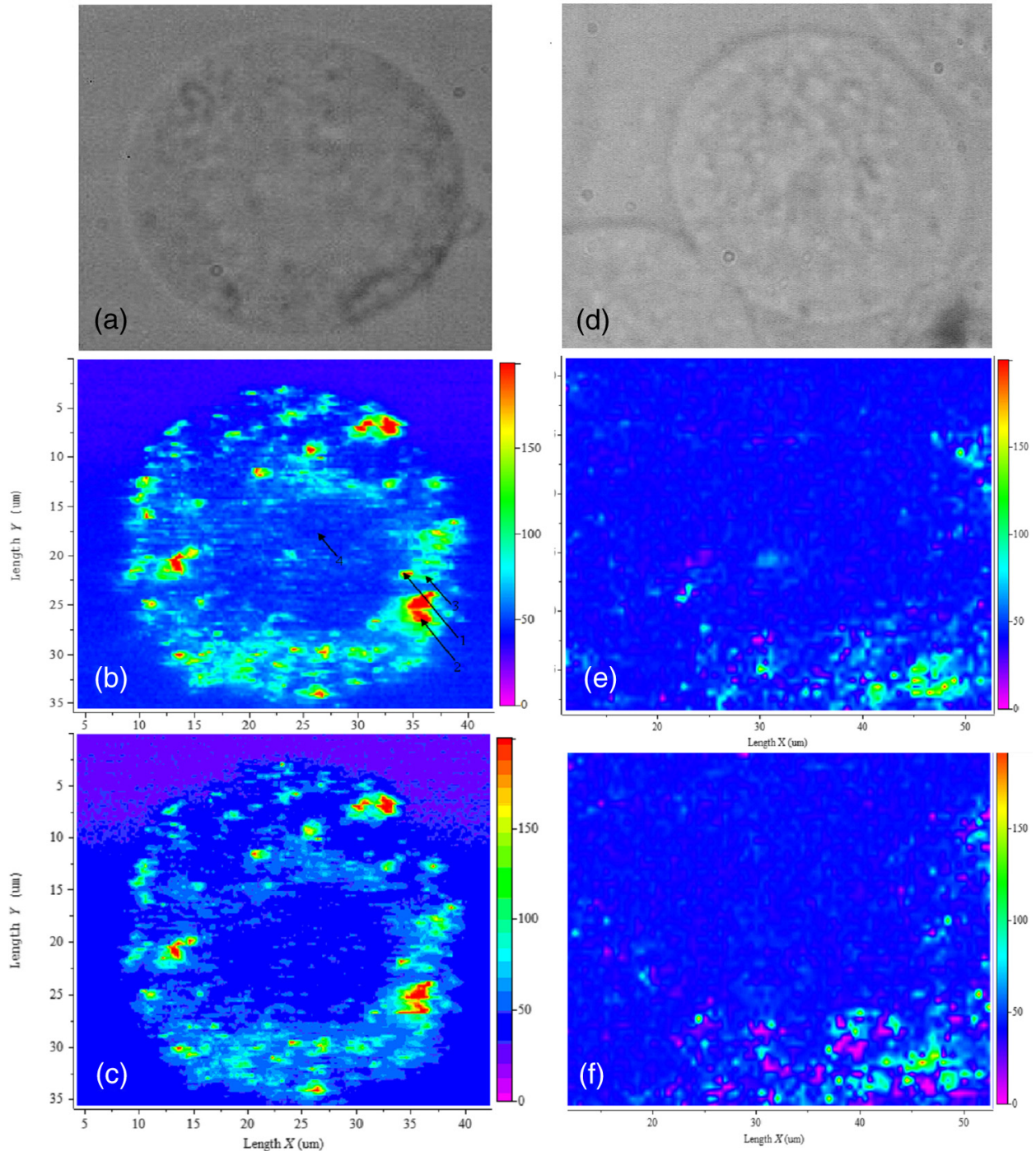

Fig. 4 White light and SERS images of cancer (A431) and normal (NHBE) cells. (a) White light image of an A431 cancer cell following incubation with 30-nm anti-EGFR-labeled AuNPs; (b) SERS image of the cancer cell obtained by an intensity map of the $1583 \mathrm{~cm}^{-1}$ peak $(C=0$ stretch in aspartic acid or glutamic acid); (c) SERS image of the cancer cell obtained by an intensity map of the $1450 \mathrm{~cm}^{-1}$ peak $\left(\mathrm{CH}_{2}\right.$ deformation/bending mode in proteins); (d) White light image of an NHBE normal cell with 30-nm anti-EGFR-labeled AuNPs attached; (e) SERS image of the normal cell obtained by an intensity map of the $1583 \mathrm{~cm}^{-1}$ peak; (f) SERS image of the normal cell obtained by an intensity map of the $1450 \mathrm{~cm}^{-1}$ peak. The left and bottom axes show the position in microns, the right axis indicates the signal intensity in CPS. 


\subsection{Raman Spectra of Cancerous and Normal Cells}

A $0.5 \mathrm{~mL}$ of $10^{6}$ per mL A431 and NHBE cells was pipetted into glass bottom Petri dishes and left to attach overnight (for A431 cells) or for 2 days (for NHBE cells) in an incubator $\left(5 \% \mathrm{CO}_{2}\right.$, $37^{\circ} \mathrm{C}$ ). $0.5 \mathrm{~mL}$ of the anti-EGFR-tagged AuNPs, at a concentration of $5 \times 10^{11}$ per mL, was added to the cells 30 min prior to the Raman experiments.

Raman spectra were collected by a point mapping scheme, with a step size of $0.3 \mu \mathrm{m}$ along $x$ and $y$. At each point, spectra are collected for $1 \mathrm{~s}$, averaged over two scans. Figure 3 shows the Raman spectra following excitation at four locations of the cell, indicated in Fig. 4(a). All peaks in the spectra are due to SERS. The simple Raman spectrum without SERS is basically the same as the background noise (spectra 4). It is clear that the SERS spectra collected at different locations vary. Figure 4(b) is a SERS image obtained by mapping the intensity of the $1583 \mathrm{~cm}^{-1}$ peak, while Fig. 4(c) is a SERS image obtained by mapping the intensity of the $1450 \mathrm{~cm}^{-1}$ peak.

The SERS images exhibit a number of interesting features [Figs. 4(b) and 4(c)]. First, there is no enhancement in the nucleus $(\mathrm{Nu})$. The signal from within the nucleus shows a featureless background. Second, SERS spectra are observed in the areas near the surface and within the cell membrane. This observation can be reconciled with the fact that the EGFR is recycled by the cell through the process of endocytosis. Furthermore, electron micrograph images presented in supplemental Fig. 5 of Ref. 13 reveal AuNPs within endosomes of cells tagged with AuNPs. Moreover, the Raman spectra are different in the four spots indicated in Fig. 3. This implies that the environment in the immediate vicinity of the AuNPs differs. Within endosomes, the EGFR is broken down into protein fragments which would produce significantly different spectra. When the image of any vibrational mode (e.g., 1450 or $1583 \mathrm{~cm}^{-1}$ ) in a given Raman spectrum is mapped, one observes little change in the Raman image (i.e., its spatial intensity distribution). While we have presented only two examples, this observation appears to be generally true. Selection of any other mode in a given spectra gives rise to a similar image, implying that the Raman image is consistent with the distribution of nanoparticle aggregates.

Table 2 lists the observed Raman shifts for each of points 1 to 3 in Fig. 3. Most of the peaks are common to anti-EGFR, as listed in Table 1. There are additional peaks which can be mostly attributed to the appearance of tyrosine and tryptophan residues, and $\mathrm{C}-\mathrm{C}$ and $\mathrm{C}=\mathrm{O}$ stretches of amino acids. New peaks appear at points internal to the cell, where the lysozyme would begin breakdown of the protein, freeing those that are already bound to the nanoparticle to make an even stronger connection and undergo reorientation. The conclusion can be drawn that there is a

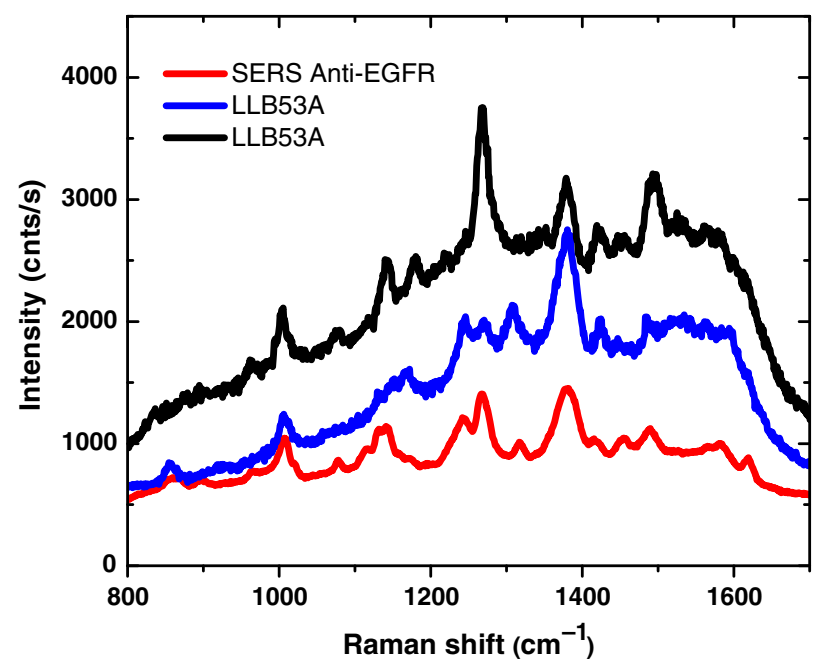

Fig. 5 SERS spectra reproduced from Fig. 2 compared with spectra (two sample scans, LLB53A) obtained from the aggregate outside the NHBE cell shown in Fig. 4(d). The spectra are very similar. LLB53A refers to the sample nomenclature used in our lab (LL-Leanne Lucas' book B, page 53 sample A). The spectral signature of the anti-EGFR antibody is present outside the cell, confirming that the nanoparticles retain their anti-EGFR coating. 
Table 2 Peak positions, relative strength-ST, and tentative assignments for the SERS spectra in the vicinity of nanoparticles, located within or on the surface of A431 cells. Peak positions obtained from Lorentzian fits (very strong-vs, strong-s, moderate-m, weak-w, and very weak-vw). Trp-tryptophan, Tyr-tyrosine, Phe-phenylalanine, Arg-arginine, and Pro-proline.

\begin{tabular}{|c|c|c|c|c|c|c|}
\hline $\begin{array}{l}\text { Point } 1 \text { spectra } \\
\text { peaks }\left(\mathrm{cm}^{-1}\right)\end{array}$ & ST & $\begin{array}{l}\text { Point } 2 \text { spectra } \\
\text { peaks }\left(\mathrm{cm}^{-1}\right)\end{array}$ & ST & $\begin{array}{l}\text { Point } 3 \text { spectra } \\
\text { peaks }\left(\mathrm{cm}^{-1}\right)\end{array}$ & ST & Assignment \\
\hline \multirow{2}{*}{$859.0 \pm 0.3$} & w & & & & & $\mathrm{Phe}^{21}$ or $\mathrm{Tyr}^{22}$ \\
\hline & & & & $884.86 \pm 0.07$ & vs & $\begin{array}{l}\text { Trp, ring breathing mode, or } \\
\mathrm{CH}_{2} \text { proteins }^{20}\end{array}$ \\
\hline $920.8 \pm 0.2$ & $\mathrm{~m}$ & & & & & $\mathrm{C}-\mathrm{C}$ stretch of proring ${ }^{20}$ \\
\hline $938.4 \pm 1.6$ & w & & & & & C-C stretch ${ }^{20}$ \\
\hline \multirow[t]{5}{*}{$981.3 \pm 0.1$} & s & & & & & Trp, Tyr, ring deformation \\
\hline & & & & $1000.6 \pm 0.6$ & w & Phe, ring stretch ${ }^{20}$ \\
\hline & & $1020.3 \pm 0.1$ & $\mathrm{~m}$ & & & Unknown \\
\hline & & $1050.1 \pm 0.1$ & $\mathrm{~m}$ & $1044.6 \pm 0.3$ & $\mathrm{~m}$ & Trp, ring stretch ${ }^{23}$ \\
\hline & & & & $1067 \pm 1$ & & $\operatorname{Arg}^{21}$ \\
\hline $1133.9 \pm 0.5$ & w & & & & & $\begin{array}{l}\text { C-C skeletal stretch } \\
\text { transconformation }{ }^{20}\end{array}$ \\
\hline $1149.4 \pm 0.7$ & w & & & & & $\mathrm{C}-\mathrm{N}$ stretch $^{20}$ \\
\hline \multirow{2}{*}{$1163 \pm 1$} & w & & & & & $\mathrm{Tyr}^{20}$ \\
\hline & & $1168.3 \pm 0.1$ & s & & & Tyr $r^{20}$ \\
\hline \multirow{2}{*}{$1218.1 \pm 0.2$} & s & $1215.0 \pm 0.5$ & w & & & Trp, Tyr ring deformation ${ }^{23}$ \\
\hline & & $1247.6 \pm 0.6$ & w & & & Amide III \\
\hline \multirow[t]{5}{*}{1273} & w & 1273 & w & $1269.2 \pm 0.3$ & $\mathrm{~m}$ & Tyr, ring deformation \\
\hline & & & & $1311.9 \pm 0.2$ & $s$ & \\
\hline & & $1330.2 \pm 0.4$ & w & & & Trp, Tyr ring stretch ${ }^{23}$ \\
\hline & & $1347.7 \pm 0.7$ & w & & & Unknown \\
\hline & & & & $1356.6 \pm 0.1$ & $\mathrm{~s}$ & Trp, ring stretch ${ }^{23}$ \\
\hline $1384.3 \pm 0.8$ & w & & & & & Tyr, ring stretch \\
\hline $1376.7 \pm 0.3$ & & & & & & $\operatorname{Arg}^{21}$ \\
\hline 1415 & w & $1415.5 \pm 0.9$ & w & & & $\mathrm{COO}^{-}$ \\
\hline $1458.8 \pm 0.5$ & $\mathrm{~m}$ & $1449.26 \pm 0.06$ & vs & & & $\begin{array}{l}\mathrm{CH}_{2} \text { deformation } / \mathrm{C}=\mathrm{C} \\
\text { bending mode of phe }\end{array}$ \\
\hline \multirow[t]{5}{*}{$1477.4 \pm 0.1$} & vs & & & $1480.3 \pm 0.4$ & w & $\mathrm{C}=\mathrm{N}$ stretch ${ }^{20}$ \\
\hline & & & & $1496.6 \pm 0.3$ & w & Unknown \\
\hline & & & & $1506.1 \pm 0.6$ & w & $\mathrm{N}=\mathrm{H}$ bending ${ }^{20}$ \\
\hline & & $1514.5 \pm 0.1$ & $S$ & & & Unknown \\
\hline & & $1525.1 \pm 0.5$ & $\mathrm{~m}$ & & & Unknown \\
\hline \multirow[t]{2}{*}{$1552.9 \pm 0.3$} & s & $1545.3 \pm 0.6$ & w & $1554.6 \pm 0.9$ & w & $\mathrm{C}=\mathrm{C}$ Trp stretch ${ }^{20}$ \\
\hline & & $1563.7 \pm 0.6$ & w & & & Trp, Tyr, ring stretch ${ }^{23}$ \\
\hline
\end{tabular}


Table 2 (Continued).

\begin{tabular}{|c|c|c|c|c|c|c|}
\hline $\begin{array}{l}\text { Point } 1 \text { spectra } \\
\text { peaks }\left(\mathrm{cm}^{-1}\right)\end{array}$ & ST & $\begin{array}{l}\text { Point } 2 \text { spectra } \\
\text { peaks }\left(\mathrm{cm}^{-1}\right)\end{array}$ & ST & $\begin{array}{l}\text { Point } 3 \text { spectra } \\
\text { peaks }\left(\mathrm{cm}^{-1}\right)\end{array}$ & ST & Assignment \\
\hline $1582.05 \pm 0.09$ & vs & & & & & Asp, Glu $C=0$ \\
\hline \multirow[t]{2}{*}{$1600.27 \pm 0.08$} & vs & $1601 \pm 2$ & w & & & Trp, Tyr ring stretch ${ }^{23}$ \\
\hline & & $1622.6 \pm 0.3$ & w & & & $\mathrm{C}=\mathrm{C}$ stretch Trp, Tyr \\
\hline
\end{tabular}

rearrangement of the amino acids upon endocytosis to make tryptophan and tyrosine residues, which both contain ring systems, more prominent in the spectra.

One can estimate the enhancement by considering the Raman signal generated by a bulk sample of anti-EGFR and the reduction in the number of anti-EGFR molecules contained within an AuNP aggregate. The numerical value of the enhancement factor is determined by comparing the Raman intensity per molecule in the presence or absence of nanoparticles with all other conditions being constant (e.g., laser power). Its value is given by $E=$ $\left(I_{\text {SERS }} / I_{\text {normal }}\right) \times\left(N_{\text {normal }} / N_{\text {SERS }}\right)$, where $N_{\text {SERS }}$ is the number of analyte molecules in the illuminated volume in the presence of nanoparticles and $N_{\text {normal }}$ is the number of molecules in the illuminated volume in the absence of nanoparticles. We estimate that the enhancement ranges from $10^{4}$ to $10^{7}$.

Figure 4(d) shows the optical image of an NHBE cell. Raman images of the $1583 \mathrm{~cm}^{-1}$ [Fig. 4(e)] and $1450 \mathrm{~cm}^{-1}$ [Fig. 4(f)] peaks show little or no enhancement over the entire cell. The only exception is a region in the lower right of the Raman images, which is outside the cell [Figs. 4(e) and 4(f)].

The optical image [Fig. 4(d)] clearly shows an aggregate of AuNPs, adhering to the Petri dish, that was not removed by the washing process. The spectra (Fig. 5) are very similar to the SERS anti-EGFR spectra of Fig. 2. The similarity of the spectra indicates that the nanoparticles retain their anti-EGFR coating. Other than this artifact, the Raman images and spectra are featureless and weak.

Figure 6 is a plot of the Raman spectra from the cells in Figs. 7(a) and 7(d). These spectra are completely featureless and are comparable with the background intensity of the cell nucleus spectrum (Fig. 3, point 4). Figure 7 shows the control experiments with A431 cells incubated with bare AuNPs [Fig. 7(a)] or nonspecific polyclonal antibody-tagged AuNPs [Fig. 7(d)].

Figure 7(b) shows the SERS intensity map at $1450 \mathrm{~cm}^{-1}$ of the cells incubated with the bare AuNPs, while Fig. 7 (c) is the intensity map at $1583 \mathrm{~cm}^{-1}$. The maps are very similar. The SERS

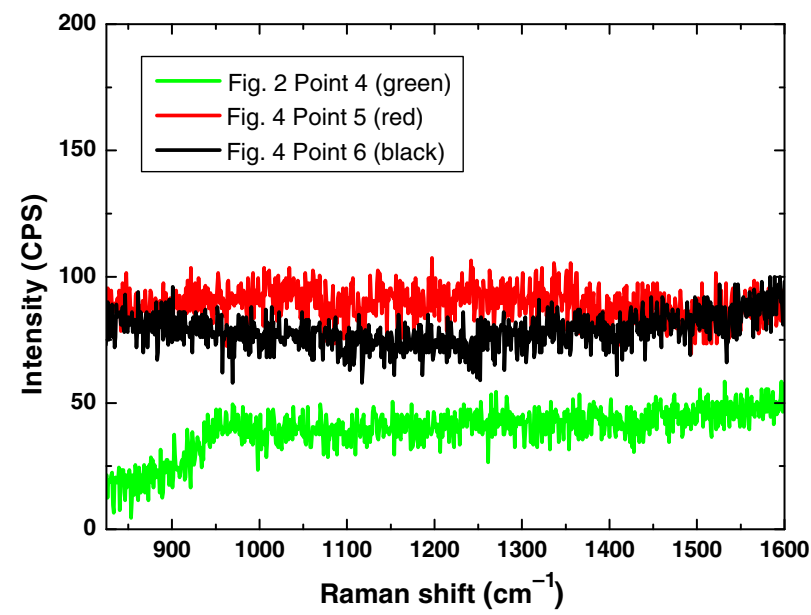

Fig. 6 SERS spectra obtained from A431 cells incubated with bare AuNPs (red) and nonspecific antibody-tagged AuNPs (black)-Fig. 7 compared with the signal obtained from the cell nucleus [Fig. 4(b), Point 4, green]. The spectra are featureless and intensities are similar. 

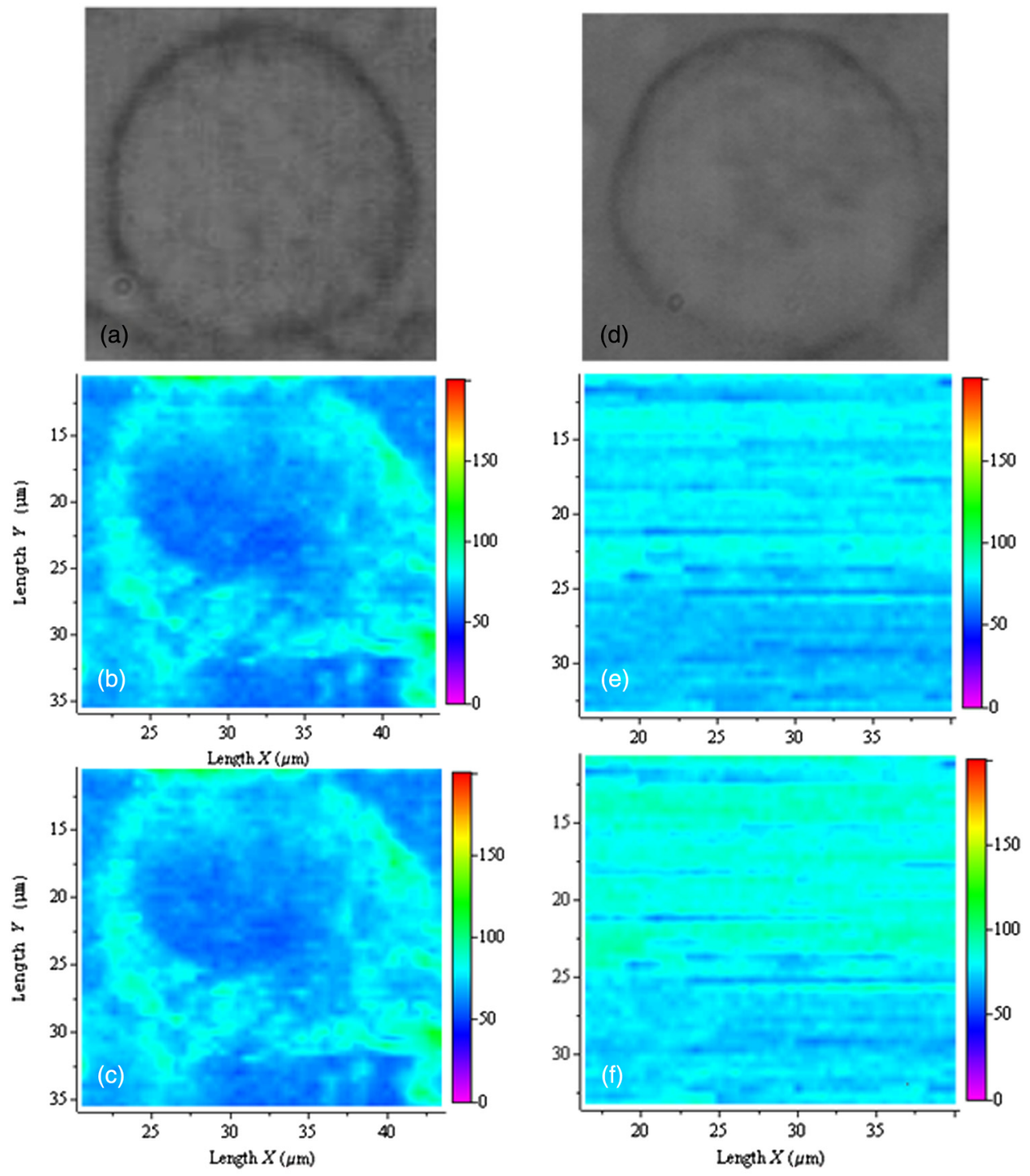

Fig. 7 White light and SERS images of A431 cancer cells. (a) White light image of an A431 cell incubated with untagged AuNPs; (b) SERS intensity map of an A431 cell at the $1450 \mathrm{~cm}^{-1}$ peak; (c) SERS intensity map of an A431 cell at the $1583 \mathrm{~cm}^{-1}$ peak; (d) white light image of an A431 cell incubated with IgG nonspecific antibody-tagged AuNPs; (e) SERS intensity map of an A431 cell at the $1450 \mathrm{~cm}^{-1}$ peak; (f) SERS intensity map of an A431 cell at the $1583 \mathrm{~cm}^{-1}$ peak. The intensities of $[(b)-(c)$ and $(e)-(f)]$ are low. Cell position is indicated by the left and bottom axes in microns, and signal intensity in CPS is indicated by the right axis.

intensity map at $1450 \mathrm{~cm}^{-1}$ for the nonspecific antibody-tagged AuNPs is shown in Fig. 7(e) and the $1583 \mathrm{~cm}^{-1}$ map is shown in Fig. 7(f). These maps are also similar, however, the spectrum at $1583 \mathrm{~cm}^{-1}$ seems to have slightly higher background intensity. The reduced intensity of the normal cell spectra [Figs. 6, 7(b)-7(c), and 7(e)-7(f)] as compared with the intensities obtained from the anti-EGFR functionalized A431 cancer cells [Figs. 3 and 4(b)-4(c)] corresponds well to expectations.

\section{Conclusions}

We have targeted EGFR in EGFR-overexpressing cells of the A431 human epidermoid carcinoma cell line (one million per milliliter) and in NHBE cells by binding anti-EGFR-tagged 30-nm AuNPs. We observed dramatic SERS effects in the A431 cells. As expected, control 
experiments with NHBE cells and with bare or nonspecific antibody-tagged AuNPs bound to A431 cells produced insignificant SERS enhancement. The spectra are associated with residues which have functional groups which associate with gold, including the sulfur containing cysteine and nitrogen containing histidine and tryptophan. Aspartic and glutamic acids would also be expected to adsorb when deprotonated, as would tyrosine through the oxygen atom side group following the loss of hydrogen. Evidence for rearrangement of these amino acids upon uptake into lysosome and endosomes is evident when comparing the SERS spectra of anti-EGFR-coated nanoparticles with spectra from cells. Tyrosine and tryptophan residues dominate.

Raman signal enhancements are observed for A431 cells and reflected the AuNP distribution. AuNPs are located on the surface as well as on the interior of the cell, while being excluded from the nucleus. The EGFR is recycled by the cell through the process of endocytosis and is not found in the nucleus. Moreover, EGFR is known to distribute nonuniformly on the cell surface. Thus, the SERS map is consistent with the expected distribution of EGFR. The SERS enhancement factor estimated from our data is between $10^{4}$ and $10^{7}$. Studies are underway to optimize the AuNP size and laser excitation wavelength. The NHBE cells do not show signal enhancements. A contrast factor of at least 100:1 is estimated, which means that a SERS image should have orders of magnitude more contrast than a typical fluorescence image or one generated by white light scattering from nanoparticles. A SERS approach to imaging EGFR distribution is, therefore, very promising.

\section{Acknowledgments}

We are indebted to the Natural Science and Engineering Research Council of Canada, the Canada Foundation for Innovation, Canadian Institute for Health Research, National Cancer Institute of Canada, and the Canadian Dermatology Foundation for financial support. We are also indebted to Drs. B. Frisken and N. Forde, both of Simon Fraser University (SFU) Physics, for making their lab available during the sabbatical of $\mathrm{KCH}$. In particular, $\mathrm{KCH}$ wishes to acknowledge the help of Dr. Frisken and her students (David Lee and Rasoul Narimani) for helping with the Dynamic Light Scattering experiment and for providing office space during his sabbatical at SFU. KCH and LL would like to thank Dr. Forde, her students, and postdocs, especially Astrid van der Horst, Suzana Kovacic, and Laleh Samii, for their willingness to help with experiments and equipment (including UV-Vis spectrometer). $\mathrm{KCH}$ is grateful also to XKC for his tremendous support during his 6-month sabbatical term at SFU.

\section{References}

1. B. Ozanne et al., "Over-expression of the EGF receptor is a hallmark of squamous cell carcinomas," J. Pathol. 149, 9-14 (1986).

2. R. E. Sobol et al., "Epidermal growth factor receptor expression in human lung carcinomas defined by a monoclonal antibody," J. Natl. Cancer Inst. 79, 403-407 (1987).

3. T. A. Libermann et al., "Expression of epidermal growth factor receptors in brain tumors," Cancer Res. 44, 753-760 (1984).

4. A. L. Harris et al., "Epidermal growth factor receptors: a marker of early relapse in breast cancer and tumor state progression in bladder cancer; interactions with neu," Cancer Cells 7, 353-357 (1989).

5. F. Hendler et al., "Increased EGF receptors and the absence of alveolar differentiation marker predict a poor survival in lung cancer," Proc. Am. Soc. Clin. Oncol. 8, 223 (1989).

6. J. R. C. Sainsbury et al., "Presence of epidermal growth factor receptor as an indicator of poor prognosis in patients with breast cancer," Clin. Pathol. 38, 1225-1228 (1985).

7. A. Eisbruch et al., "Analysis of the epidermal growth factor receptor gene in fresh human head and neck tumors," Cancer Res. 47, 3603-3605 (1987).

8. E. Hsu et al., "A far-red fluorescent contrast agent to image epidermal growth factor receptor expression," Photochem. Photobiol. 79, 272-279 (2004). 
9. J. Aaron et al., "Plasmon resonance coupling of metal nanoparticles for molecular imaging of carcinogenesis in vivo," J. Biomed. Opt. 12, 034007 (2007).

10. K. Kneipp, M. Moskovits, and H. Kniepp, Surface Enhanced Raman Spectroscopy: Physics and Applications, Springer-Verlag, New York (2006).

11. M. Y. Sha et al., "SERS nanoparticles: a new optical detection modality for cancer diagnosis," Nanomedicine 2, 725-734 (2007).

12. J. C. Y. Kah et al., "Early diagnosis of oral cancer based on the surface plasmon resonance of gold nanoparticles," Int. J. Nanomed. 2, 785-798 (2007).

13. X. Qian et al., "In vivo tumor targeting and spectroscopic detection with surface-enhanced Raman nanoparticle tags," Nat. Biotechnol. 26, 83-90 (2008).

14. S. Keren et al., "Noninvasive molecular imaging of small living subjects using Raman spectroscopy," Proc. Natl. Acad. Sci. U. S. A. 105, 5844-5849 (2008).

15. J.-H. Kim et al., "Nanoparticles probes with surface enhanced Raman spectroscopic tags for cellular cancer targeting," Anal. Chem. 78, 6967-6973 (2006).

16. L. J. Lucas et al., "Imaging EGFR distribution using surface-enhanced Raman spectroscopy," Proc. SPIE 7192, 719206 (2009).

17. J. Turkevich, P. C. Stevenson, and J. Hillier, "A study of the nucleation and growth processes in the synthesis of colloidal gold," Discuss. Faraday Soc. 11, 55-75 (1951).

18. G. Frens, "Controlled nucleation for the regulation of particle-size in monodisperse gold suspensions," Nat.-Phys. Sci. 241, 20-22 (1973).

19. X. Huang et al., "Cancer cells assemble and align gold nanorods conjugated to antibodies to produce highly enhanced, sharp and polarized surface enhanced Raman spectra," Nano Lett. 7(6), 1591-1597 (2007).

20. Z. Movasaghi, S. Rehman, and I. U. Rehman, "Raman spectra of biological tissue," Appl. Spectrosc. Rev. 42(5), 493-541 (2007).

21. J. De Gelder et al., "Reference database of Raman spectra of biological molecules," J. Raman Spectrosc. 38, 1133-1147 (2007).

22. R. P. Kengne-Momo et al., "Protein interactions investigated by the Raman spectroscopy for biosensor applications," Int. J. Spectrosc. 2012, 462901 (2012).

23. E. S. Grabbe and R. P. Buck, "Surface-enhanced Raman spectroscopic investigation of human immunoglobin G adsorbed on a silver electrode," J. Am. Chem. Soc. 111, 83628366 (1989).

Leanne J. Lucas graduated from Dalhousie University with a Bachelor of Science, Honors Coop in chemistry (2010), and a Master of Science in physics and atmospheric science (2013). Her research interests include Raman spectroscopy, proteomics, nonconventional cancer diagnosis and treatment methods, and renewable energy. She was the first African Nova Scotian to receive a graduate degree from the Department of Physics and Atmospheric Science (2013). She currently works at Maxxam Analytics in Bedford, Nova Scotia.

Xiaoke K. (Michael) Chen is a senior lecturer at Simon Fraser University. He received his $\mathrm{PhD}$ degree from the City University of New York in 1991. He is the author of 42 journal articles. His current research interests are Raman spectroscopy and physics education.

Aaron J. Smith is a scientist at Tesla Motors. He received his $\mathrm{PhD}$ degree in physics from Dalhousie University in 2012. His current focus is on advance lithium-ion battery testing and analysis.

Mladen Korbelik is a distinguished scientist at the British Columbia Cancer Agency (Vancouver, British Columbia) and professor at the Pathology and Laboratory Medicine Department, University of British Columbia. He has authored around 150 journal papers or book chapters. His research interests include the use of photodynamic therapy for treatment of solid malignant tumors and the associated inflammatory/immune host response, vaccines, and other forms of cancer immunotherapy.

Haishan Zeng is a distinguished scientist at the British Columbia Cancer Agency and a professor of dermatology, pathology, and physics at the University of British Columbia. His 
research has been focused on developing optical imaging and spectroscopy techniques for improving early cancer detection. He has published over 123 refereed journal papers, 1 book, 8 book chapters, and has 20 granted patents.

Patrick W. K. Lee is a professor and Cameron Chair in Basic Cancer Research at Dalhousie University. He received his $\mathrm{PhD}$ degree in biochemistry from the University of Alberta in 1978. $\mathrm{He}$ is the author of more than 100 journal articles. His current research focuses on oncolytic viruses, oncogenes, and tumor suppressors.

Kevin Cecil Hewitt is an associate professor of physics at Dalhousie University. He received his BSc degree in physics and biology from the University of Toronto and a PhD degree in physics from Simon Fraser University, in 2000. He is the author of more than 30 journal articles and has written 1 book chapter. His current research interests include Raman spectroscopy, holography, and simulated Raman spectroscopy. 